\title{
Croissance et prise de l'acide glutamique par les bactéries du genre Streptococcus en fonction de la source d'énergie
}

\author{
A. BENATEYA, P. BRACQUART, J.-Y. LE DEAUT et G. LINDEN \\ Laboratoire de Biochimie appliquée, Université de Nancy I \\ B.P. 239 - 54506 Vandouvre-les-Nancy, France
}

\section{Résumé}

La fermentation du lactose du lait par les bactéries lactiques, essentiellement les genres Streptococcus et Lactobacillus, est primordiale en Industrie Laitière. Depuis une dizaine d'années, les systèmes de transport et d'hydrolyse des glucides par ces microorganismes ont été étudiés. Relativement aux autres bactéries du même genre, il existe peu de données sur le métabolisme glucidique chez Streptococcus thermophilus.

La présente étude a porté sur l'effet de la source d'énergie sur la croissance et la prise de l'acide glutamique chez différentes espèces de streptocoques (35 souches appartenant à 14 espèces). Nous avons pu constater l'originalité de $S$. thermophilus : le lactose est un meilleur substrat que le glucose aussi bien pour le transport actif que pour la croissance de la bactérie. Tous les autres streptocoques testés sauf peut-être $S$. salivarius préfèrent le glucose ou donnent des résultats similaires avec les deux glucides. L'adaptation préalable des streptocoques du groupe $\mathrm{N}$ au lactose les rend plus aptes à fermenter ce diholoside.

Mots clés : Streptococcus - Prise de l'acide glutamique - Lactose - Glucose - Adaptation.

\section{Summary}

Influence of energy source on the growth and glutamic acid uptake by Streptococcus genus

Milk lactose fermentation by lactic acid bacteria, especially Streptococcus and Lactobacillus genera is important in the Dairy Industry. For about ten years, the systems of sugar transport and subsequent hydrolysis by these microorganisms have been studied. With respect to the other bacteria of the same genus, very few data on carbohydrate metabolism in Streptococcus thermophilus are available whereas a great amount of research attention has been given to this fermentation by the group $\mathrm{N}$ streptococci.

The present study examines the influence of the energy source on the growth and glutamic acid uptake by different species of streptococci ( 35 strains from 14 species) 
and their adaptation to glucose and lactose. Our experiments show the special behaviour of $S$. thermophilus: lactose is seen to be better substrate than glucose for both bacterial growth and active transport. All the other streptococci tested, except perhaps $S$. salivarius, prefer glucose or use both sugars in the same way. A previous adaptation of group N streptococci enhances the use of this disaccharide. According to these data, we have classified the streptococci studied in three groups.

Key words : Streptococcus - Glutamic acid uptake - Lactose - Glucose - Adaptation.

\section{Introduction}

La fermentation du lactose du lait par les bactéries des genres Streptococcus et Lactobacillus présente un grand intérêt pour l'Industrie laitière. L'acide lactique produit par cette réaction contribue à la flaveur, la texture et le maintien de la qualité du produit fermenté. Les études sur le métabolisme des glucides ont jusqu'à présent été développées essentiellement chez les streptocoques du groupe $\mathrm{N}$ (Bissett et Anderson, 1974 ; LeE, 1974 ; McKay et al., 1969, 1970, 1971 ; Thomas et al., 1980 ; Thompson et Thomas, 1977 ; Thompson, 1978 ; Thompson et al., 1978 ; Thompson, 1979 ; Thompson, 1980 ; Thompson et Chassy, 1981).

Le transport des glucides, en particulier le lactose, à travers la membrane des bactéries de ce groupe est en général assuré par le système phosphotransférase phosphoénolpyruvate dépendant (PEP-PTS) (MCKAY et al., 1969, Thomas et al., 1980). Le mécanisme de ce système a été mis en évidence pour la première fois chez $E$. coli par Kundig et al. (1964). Lors de son transport le lactose est phosphorylé puis hydrolysé par une phospho- $\beta$-galactosidase $(\mathrm{P}-\beta$-gal) en glucose et galactose-6-phosphate qui sont respectivement métabolisés selon les voies d'Embden-Meyerhof-Parnas et du Tagatose-6-phosphate (LAWrence et al., 1976). En outre, LEE et al. (1973) ont montré que certains streptocoques, notamment $S$. cremoris HP et $S$. lactis $\mathrm{C} 2$, sont capables de transporter le glucose par un système de type perméase dépendant de 1'ATP (Boos, 1974). D'autres auteurs (KASHKET et WILSON, 1972 a, 1972 b ; KASHKET et al., 1980 ; KASHKET, 1981) ont montré que chez $S$. lactis ATCC 7962 le transport actif du thiométhyl- $\beta$-galactoside est couplé à un flux de protons dont le gradient est maintenu par une pompe électrogénique. La valinomycine induit le transport actif du galactoside, elle catalyse l'uniport net d'ions $\mathrm{K}^{+}$et augmente la conductance de protons $\mathrm{H}^{+}$à l'intérieur de la cellule. Ces observations sont en accord avec les hypothèses chemiosmotiques de Mitchell (1966).

Contrairement aux streptocoques du groupe $\mathrm{N}$, le métabolisme des glucides chez $S$. thermophilus a été peu étudié et les données qui existent sont encore contradictoires. HEMME et al. (1980 a et 1980 b) ont trouvé à la fois une activité $\beta$-galactosidase et une faible activité phospho- $\beta$-galactosidase, par contre GrEENBERG et MAHONEY (1982) et TINSON et al. (1982) ne trouvent qu'une activité $\beta$-galactosidase. Ces derniers pensent que l'activité phospho- $\beta$-galactosidase serait due à une phosphatase. Ces faits suggèrent l'absence d'un système PEP-PTS chez $S$. thermophilus remplacé par un système perméase pour le transport du lactose. Le diholoside pénétrerait donc à l'intérieur de la cellule sous forme libre avant d'être hydrolysé par une $\beta$-galactosidase en glucose et galactose (LAW et Sharpe, 1978). Le glucose est converti en acide lactique selon la voie d'Embden- 
Meyerhof-Parnas alors que le galactose serait excrété dans le milieu de culture (O'Leary et WoychicK, 1976; Thomas et Crow, 1983).

AKPEMADo (1981) a montré que le transport des acides aminés à chaîne ramifiée chez $S$. thermophilus est un système actif et que le lactose est une excellente source d'énergie pour ce phénomène. De plus le Bergey's Manual (DerBeL et Seeley, 1974) indique bien l'utilisation préférentielle de ce disaccharide par cette espèce. A travers ce travail nous avons essayé d'étudier le potentiel énergétique de la prise de l'acide glutamique chez un grand nombre de bactéries du genre Streptococcus. Cet acide aminé a été choisi car divers travaux ont montré que c'est l'acide aminé le plus exigé pour une bonne croissance chez la plupart des streptocoques (LAW et al., 1976; Mills et Thomas, 1981 ; Bracouart, 1985).

\section{Matériel et méthodes}

\section{- Bactéries étudiées}

Les espèces et souches étudiées sont indiquées dans le tableau 1.

\section{- Croissance des bactéries}

Le milieu utilisé pour la croissance des streptocoques est le milieu Tryptose Protéose Peptone à l'extrait de levure (TPPY) (BRACQUART, 1981) avec comme source d'énergie le glucose ou le lactose. Un volume de $80 \mathrm{ml}$ est réparti dans des ballons de $100 \mathrm{ml}$. L'inoculum de $2 \mathrm{ml}$ est réalisé à partir d'une bactérie ayant subi deux précultures successives de $13 \mathrm{~h}$ dans le même milieu à la température optimale de croissance. Les températures utilisées sont les suivantes :

- S. thermophilus : $42^{\circ} \mathrm{C}$;

- streptocoques du groupe $\mathrm{N}: 30^{\circ} \mathrm{C}$;

- S. cremoris : $25^{\circ} \mathrm{C}$;

- streptocoques fécaux et autres streptocoques : $37^{\circ} \mathrm{C}$.

La croissance bactérienne est suivie sur le milieu lactosé d'une part et sur le milieu glucosé d'autre part pour les cellules adaptées à l'un et l'autre glucide. Elle est estimée par lecture de la densité optique (D.O.) au spectromètre d'absorption moléculaire à une longueur d'onde de $650 \mathrm{~nm}$.

\section{- Prise de l'acide glutamique}

La culture sur milieu lactosé ou glucosé est arrêtée en phase exponentielle, quand la D.O. mesurée à $650 \mathrm{~nm}$ est égale à 0,5 . Le $\mathrm{pH}$ atteint alors des valeurs comprises entre 4,8 et 5,2 selon les espèces et les souches d'une même espèce. Les cellules sont centrifugées à $5000 \mathrm{~g}$ pendant $5 \mathrm{~min}$ à $10^{\circ} \mathrm{C}$ puis lavées deux fois avec $10 \mathrm{ml}$ de tampon Tris- $\mathrm{H}_{2} \mathrm{SO}_{4} 0,05 \mathrm{M}, \mathrm{pH} 7,0$. Le culot cellulaire obtenu après lavages et centrifugations est remis en suspension dans le même tampon. La concentration finale en bactéries est de $1 \mathrm{mg}$ de cellules en poids sec par ml d'essai par référence à une courbe étalon donnant la D.O. en fonction du poids sec de bactéries.

Un mélange de $1 \mathrm{ml}$ de suspension cellulaire et de $100 \mu 1$ de tampon ou de solution de glucide à une concentration finale de $40 \mathrm{mM}$ est incubé pendant 10 min à la température optimale de croissance de la bactérie. La réaction commence dès l'addition de $100 \mu 1$ d'une solution de L.U. ${ }^{14} \mathrm{C}$ acide glutamique 
Tableau I. - Espèces et souches étudiées - Species and strains studied

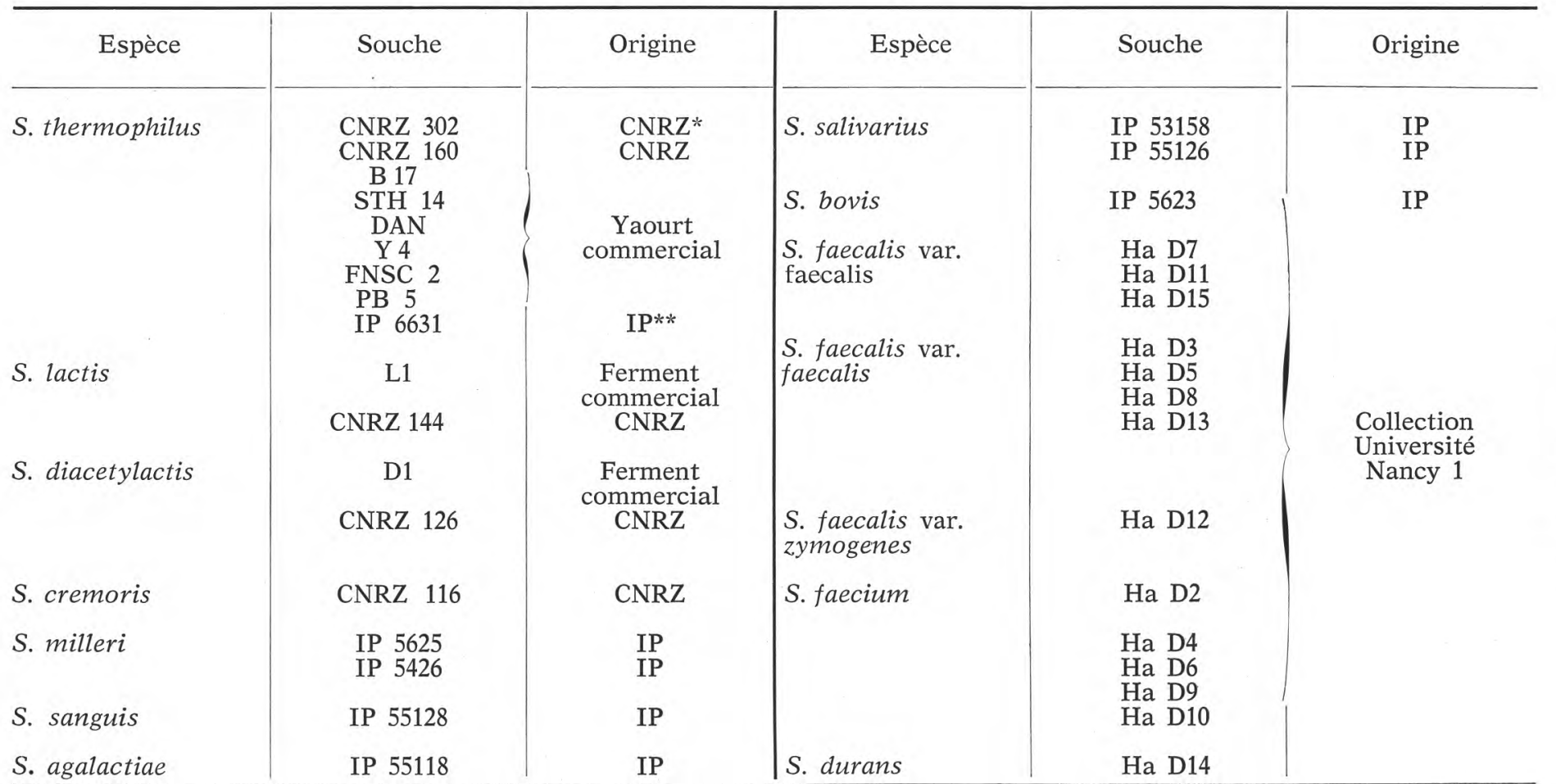

* CNRZ : Centre National de Recherches Zootechniques.

** IP : Institut Pasteur. 
$0,2 \mathrm{mM}$ dont la concentration radioactive est de $275 \mathrm{mCi} / \mathrm{mmole}$ (Amersham, Angleterre). Au bout d'une minute et $5 \mathrm{~min}, 400 \mu \mathrm{l}$ sont prélevés et filtrés sur membrane Millipore de diamètre $25 \mathrm{~mm}$ et de porosité $0,45 \mu \mathrm{m}$. Les filtres sont rapidement lavés deux fois avec $5 \mathrm{ml}$ de tampon, séchés sommairement et mis dans des fioles à compter en présence de $10 \mathrm{ml}$ de scintillateur A.C.S. (Aqueous Counting Scintillant, Amersham). Le comptage est effectué pendant $10 \mathrm{~min}$ au compteur à scintillation Intertechnique SL 4000 à enceinte réfrigérée.

Pour chaque essai $400 \mu 1$ du milieu réactionnel sont prélevés et directement comptés, donnant la quantité exacte de radioactivité présente initialement. Les essais sont réalisés deux ou trois fois.

La quantité d'acide aminé absorbée est déterminée par le nombre de coups par minute (CPM) des cellules rapportée à la quantité de radioactivité initialement présente.

\section{Résultats}

\section{A. Croissance des streptocoques}

Nous avons étudié la croissance sur milieu TPPY de 9 souches d'origines diverses appartenant à l'espèce $S$. thermophilus. Pour chaque souche, nous avons testé l'utilisation du lactose et celle du glucose en les adaptant au préalable à l'un ou l'autre glucide. Nous remarquons que toutes les souches montrent une meilleure croissance quand le milieu de culture contient du lactose indépendamment du glucide d'adaptation préalable. Cette préférence du lactose est relativement importante, en particulier chez les souches CNRZ 302, FNSC 2, Y 4 et IP 6631 ; elle se traduit par une phase de latence plus courte et une densité optique finale plus élevée. Chez $S$, thermophilus CNRZ 302 (fig. $1 \mathrm{~A}$ ) par exemple, on remarque qu'en $4 \mathrm{~h}$ d'incubation la D.O. du milieu de culture lactosé atteint 0,7 à 0,8 alors que celle des cellules cultivant sur milieu glucosé n'est que 0,1 à 0,2 ; pour un même temps de culture, les premières se trouvent en fin de phase exponentielle de croissance et les autres tout au début de la même phase. La différence de temps de latence entre la croissance sur lactose et celle sur glucose varie de $60 \mathrm{~min}$ à $180 \mathrm{~min}$ selon les souches.

La majorité des souches de $S$. thermophilus (CNRZ 302, CNRZ 160, B 17, Y 4, DAN et IP 6631) présentent une légère préférence pour le lactose quand les cellules ont été adaptées au lactose plutôt qu'au glucose. De même, sur glucose la croissance est légèrement meilleure pour les bactéries provenant de la préculture lactosée.

A l'image de $S$. thermophilus, les deux souches de $S$. salivarius montrent une croissance plus importante quand le lactose est présent comme seule source d'énergie. Les bactéries ayant été adaptées au lactose cultivent encore mieux sur lactose que sur glucose. Cependant la différence entre la croissance sur lactose et sur glucose est moins importante que celle observée pour la plupart des souches $S$. thermophilus. La différence de temps de latence $(\Delta \mathrm{tl})$ n'est que de $40 \mathrm{~min}$ et $60 \mathrm{~min}$ respectivement pour S. salivarius IP 55126 et S. salivarius IP 53158 . 


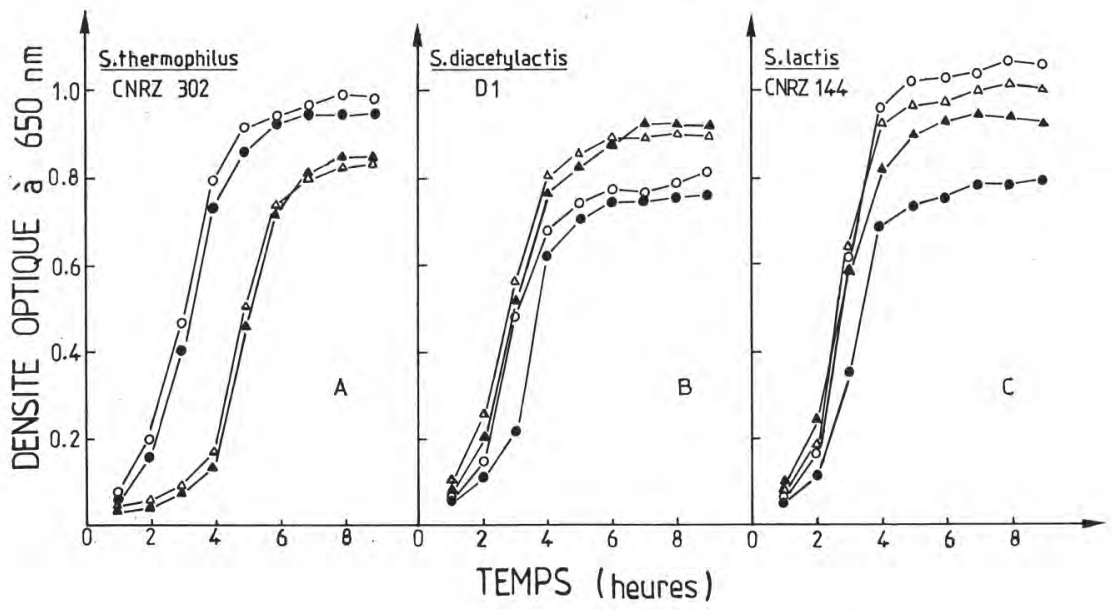

Fig. 1

Courbes de croissance sur milieu TPPY de:

Growth curves on TPPY medium of :

(A) S. thermophilus $C N R Z 302$ à $42^{\circ} C$.

(B) S. diacetylactis $D 1$ à $30^{\circ} \mathrm{C}$.

(C) S. lactis CNRZ 144 à $30^{\circ} \mathrm{C}$.

○- - Lac.Lac (adaptation au lactose, culture sur lactose).

- Glc.Lac (adaptation au glucose, culture sur lactose).

$\triangle \longrightarrow \triangle$ Lac.Glc (adaptation au lactose, culture sur glucose).

_- Glc.Glc (adaptation au glucose, culture sur glucose).

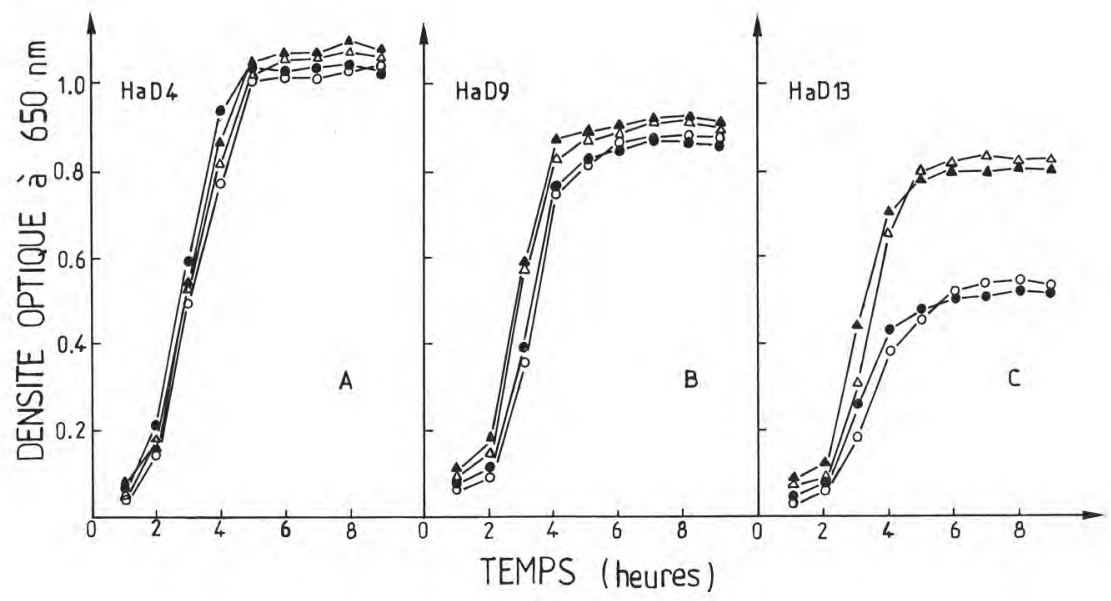

Fig. 2

Courbes de croissance sur milieu TPPY de:

Growth curves on TPPY medium of :

(A) S. faecium $\mathrm{HaD} 4$ à $37^{\circ} \mathrm{C}$.

(B) S. faecalis var liquifaciens $\mathrm{HaD5}$ à $37^{\circ} \mathrm{C}$.

(C) $\mathrm{S}$. faecalis var liquefaciens $\mathrm{HaD} 13$ à $37^{\circ} \mathrm{C}$.

○- - Lac.Lac (adaptation au lactose, culture sur lactose).

- Glc.Lac (adaptation au glucose, culture sur lactose).

$\triangle \longrightarrow \triangle$ Lac.Glc (adaptation au lactose, culture sur glucose).

—_ Glc.Glc (adaptation au glucose, culture sur glucose). 
Contrairement aux deux espèces précédentes, les streptocoques du groupe $\mathrm{N}$ montrent une utilisation préférentielle du glucose. Deux souches de $S$. lactis et deux de $S$. diacetylactis ont été étudiées et les résultats sont pratiquement similaires. De plus, l'adaptation de ces bactéries au lactose favorise leur croissance sur ce même glucide, ce qui s'observe surtout en phase exponentielle (fig. $1 \mathrm{~B}$ et $1 \mathrm{C})$.

En général, les streptocoques fécaux ne montrent pas de préférence vis-à-vis du lactose ou du glucose, la croissance est sensiblement identique sur les deux substrats pour 1'ensemble des souches (fig. 2 A). Toutefois quelques particularités sont à signaler; en effet, $S$. faecalis var. liquefaciens HaD5 (fig. $2 \mathrm{~B}$ ) tout comme $S$. faecium $\mathrm{HaD} 9$ semblent utiliser préférentiellement le glucose et se rapprochent plutôt des streptocoques du groupe N. Au contraire, S. sanguis IP 55128 , espèce appartenant au groupe «oral» (JoNES, 1978), se comporte comme les streptocoques fécaux. Quant à $S$. faecalis var. liquefaciens HaD13, elle paraît très différente des autres streptocoques de la même espèce du fait qu'elle cultive bien sur glucose alors que sur lactose, la croissance est rapidement ralentie autour de 0,4 de densité optique (fig. 2 C).

\section{B. Prise de l'acide glutamique}

Des études préliminaires nous ont montré qu'en absence de solution glucidique dans le milieu réactionnel, la prise de l'acide glutamique est quasiment nulle chez l'ensemble des espèces étudiées.

Nous exprimons nos résultats de prise de l'acide aminé en pourcentage par rapport à la quantité d'acide aminé absorbée en présence de glucose par les cellules adaptées au glucose, qui sert de référence $(100 \%)$. Ceci nous permet une comparaison plus facile des résultats entre les différentes bactéries.

\section{S. thermophilus et $S$. salivarius}

Le tableau 2 indique le pourcentage de prise de l'acide glutamique en présence de glucose ou de lactose par diverses souches de $S$. thermophilus et $S$. salivarius adaptées à l'un ou l'autre glucide. Les résultats montrent que toutes ces bactéries effectuent une meilleure perméation de l'acide aminé avec le lactose comme source d'énergie quel que soit le glucide d'adaptation préalable. Néanmoins, pour les bactéries provenant du milieu lactosé, deux catégories sont à distinguer :

- les souches qui utilisent nettement mieux le lactose, ce sont essentiellement des souches $S$. thermophilus CNRZ 302, FNSC 2, DAN, STH 14 et Y 4 ;

- les souches qui montrent une activité en présence de lactose relativement moins importante, ce sont $S$. thermophilus CNRZ 160, B 17 et PB 5.

Toutes les souches de $S$. thermophilus, à l'exception de CNRZ 302 et Y 4, utilisent mieux le glucose pour transporter l'acide aminé lorsqu'elles proviennent du milieu lactosé par rapport à leur culture sur glucose.

Les deux souches de $S$. salivarius montrent également en présence de lactose une meilleure activité de perméation de l'acide glutamique mais cette préférence n'est pas aussi marquée que pour la plupart des souches de $S$. thermophilus.

\section{Streptocoques du groupe $N$}

Cultivés sur milieu glucosé, les streptocoques du groupe N prennent en général très faiblement l'acide glutamique quand le lactose est la source d'énergie 
TABLEAU II

Pourcentage de prise de l'acide glutamique par S. thermophilus et $\mathrm{S}$. salivarius en présence de lactose ou de glucose avec adaptation préalable au lactose ou au glucose

Percent of acid glutamic uptake by S. thermophilus and S. salivarius adapted on lactose or glucose using lactose or glucose as energy source

\begin{tabular}{|c|c|c|c|c|c|c|c|c|}
\hline \multirow{2}{*}{ Souche } & \multicolumn{2}{|c|}{ Glc.Lac./Glc.Glc. } & \multicolumn{2}{|c|}{ Lac.Glc./Glc.Glc. } & \multicolumn{2}{|c|}{ Lac.Lac./Glc.Glc. } & \multicolumn{2}{|c|}{ Lac.Lac./Lac.Glc. } \\
\hline & $1^{\prime}$ & $5^{\prime}$ & $1^{\prime}$ & $5^{\prime}$ & $1^{\prime}$ & $5^{\prime}$ & $1^{\prime}$ & $5^{\prime}$ \\
\hline \multicolumn{9}{|l|}{ S. thermophilus } \\
\hline $\begin{array}{l}\text { CNRZ } 302 \\
\text { FNSC } 2 \\
\text { DAN } \\
\text { STH } 14 \\
\text { Y } 4 \\
\text { CNRZ } 160 \\
\text { B 17 } \\
\text { PB 5 }\end{array}$ & $\begin{array}{l}890 \\
191 \\
182 \\
137 \\
126 \\
190 \\
235 \\
268\end{array}$ & $\begin{array}{l}450 \\
182 \\
207 \\
155 \\
139 \\
123 \\
364 \\
369\end{array}$ & $\begin{array}{r}34 \\
122 \\
153 \\
107 \\
59 \\
221 \\
112 \\
174\end{array}$ & $\begin{array}{r}19 \\
124 \\
154 \\
112 \\
68 \\
228 \\
134 \\
189\end{array}$ & $\begin{array}{l}760 \\
215 \\
284 \\
222 \\
120 \\
252 \\
151 \\
290\end{array}$ & $\begin{array}{l}360 \\
252 \\
309 \\
239 \\
191 \\
263 \\
146 \\
243\end{array}$ & $\begin{array}{r}2200 \\
176 \\
185 \\
208 \\
203 \\
113 \\
134 \\
166\end{array}$ & $\begin{array}{r}3000 \\
203 \\
200 \\
213 \\
277 \\
115 \\
109 \\
128\end{array}$ \\
\hline \multicolumn{9}{|l|}{ S. salivarius } \\
\hline $\begin{array}{lll}\text { IP } & 53 & 158 \\
\text { IP } & 55 & 126\end{array}$ & $\begin{array}{l}118 \\
188\end{array}$ & $\begin{array}{l}120 \\
147\end{array}$ & $\begin{array}{l}153 \\
191\end{array}$ & $\begin{array}{l}136 \\
149\end{array}$ & $\begin{array}{l}175 \\
215\end{array}$ & $\begin{array}{l}130 \\
147\end{array}$ & $\begin{array}{l}114 \\
112\end{array}$ & $\begin{array}{l}95 \\
98\end{array}$ \\
\hline
\end{tabular}

- Glc : Glucose ; Lac : Lactose.

- Le premier glucide correspond au milieu de culture et le second à l'essai de prise.

- The first sugar corresponds to the culture medium and the second one to the amino acid uptake. 
(tabl. 3). Au contraire, une culture préalable sur milieu lactosé permet à ces bactéries de bien utiliser ce diholoside pour transporter l'acide aminé à l'intérieur des cellules.

\section{Entérocoques}

Le tableau 4 montre que les valeurs de prise de l'acide glutamique sont plus ou moins homogènes et se situent autour de $100 \%$ pour l'ensemble des streptocoques fécaux, ce qui suggère que l'acide aminé est pratiquement pris de la même manière en présence de l'un ou l'autre glucide quelle que soit l'adaptation préalable.

Cependant, quelques exceptions sont à signaler, à savoir que les souches $S$. faecalis var. liquefaciens $\mathrm{HaD} 3$ et $\mathrm{HaD} 5$ et $S$. faecium $\mathrm{HaD} 9$ utilisent préférentiellement le glucose, ce qui est en corrélation avec les résultats de la croissance.

\section{Autres streptocoques}

L'activité de prise de l'acide glutamique par $S$. milleri, $S$. bovis et $S$. agalactiae semble plus importante en présence de glucose que de lactose (tabl. 5). Quant à $S$. sanguis, il présente des résultats similaires à ceux de la plupart des entérocoques.

\section{Discussion}

Des études antérieures chez S. lactis (RICE et al., 1978), S. agalactiae (MORAN, 1980) et $S$. thermophilus (AKPEMADO et BRACQUART, 1983) ont montré que le transport des acides aminés est assuré par un système actif. Notre étude nous a permis de constater qu'en absence de source d'énergie, le transport de l'acide glutamique est pratiquement insignifiant; le phénomène de diffusion est négligeable chez l'ensemble des espèces étudiées.

En présence de lactose, $S$. thermophilus prend plus rapidement l'acide glutamique quel que soit le glucide d'adaptation préalable. Cette préférence se retrouve également dans les courbes de croissance et se traduit par une phase de latence plus courte et une D.O. finale plus élevée. Le transport du lactose est alors exprimé constitutivement indépendamment du glucide d'adaptation préalable. Ce fait a été aussi signalé par Somkuti et Steinberg (1979).

Par ailleurs, nos résultats montrent que l'utilisation du glucose par cette espèce nécessite une phase de latence relativement importante, ce qui suggère que le système est adaptatif.

Nous avons également étudié le comportement d'autres souches de la même espèce et d'autres espèces du même genre à travers l'adaptation aux glucides et le potentiel énergétique du lactose et du glucose vis-à-vis de la prise de l'acide glutamique. Le présent travail nous a permis de classer les bactéries du genre Streptococcus étudiées en trois groupes.

Le premier groupe comporte les streptocoques qui montrent une préférence nette pour le lactose comme source d'énergie aussi bien pour la croissance que 


\section{TABLEAU III}

Pourcentage de prise de l'acide glutamique chez les streptocoques du groupe $N$ en présence de lactose ou de glucose avec adaptation préalable au lactose ou au glucose

Percent of acid glutamic uptake by the group $N$ streptococci adapted on lactose or glucose using lactose or glucose as energy source

\begin{tabular}{|c|c|c|c|c|c|c|c|c|}
\hline Souche & $1^{\prime}$ & $5^{\prime}$ & $1^{\prime}$ & $5^{\prime}$ & $1^{\prime}$ & $5^{\prime}$ & $1^{\prime}$ & $5^{\prime}$ \\
\hline S. lactis L1 & 11 & 17 & 41 & 49 & 39 & 59 & 95 & 120 \\
\hline $\begin{array}{l}\text { S. lactis } \\
\text { CNRZ } 144\end{array}$ & 52 & 56 & 62 & 69 & 71 & 76 & 114 & 110 \\
\hline $\begin{array}{l}\text { S. cremoris } \\
\text { CNRZ } 116\end{array}$ & 74 & 57 & 60 & 55 & 64 & 62 & 106 & 112 \\
\hline $\begin{array}{l}\text { S. diacetylactis } \\
\text { D1 }\end{array}$ & 335 & 248 & 175 & 226 & 161 & 210 & 92 & 92 \\
\hline $\begin{array}{l}\text { S. diacetylactis } \\
\text { CNRZ } 126\end{array}$ & 29 & 36 & 99 & 86 & 101 & 93 & 102 & 108 \\
\hline
\end{tabular}

— Glc. : Glucose ; Lac. : Lactose.

- Le premier glucide correspond au milieu de culture et de second à l'essai de prise.

- The first sugar corresponds to the culture medium and the second one to the amino acid uptake. 
TABLEAU IV

Pourcentage de prise de l'acide glutamique chez les streptocoques du groupe $D$ en présence de lactose ou de glucose avec adaptation préalable au lactose ou au glucose

Percent of acid glutamic uptake by the group D streptococci adapted on lactose or glucose using lactose or glucose as energy source

\begin{tabular}{|c|c|c|c|c|c|c|c|c|}
\hline \multirow{2}{*}{ Souche } & \multicolumn{2}{|c|}{ Glc.Lac./Glc.Glc. } & \multicolumn{2}{|c|}{ Lac.Glc./Glc.Glc. } & \multicolumn{2}{|c|}{ Lac.Lac./Glc.Glc. } & \multicolumn{2}{|c|}{ Lac.Lac./Lac.Glc. } \\
\hline & $1^{\prime}$ & $5^{\prime}$ & $1^{\prime}$ & $5^{\prime}$ & $1^{\prime}$ & $5^{\prime}$ & $1^{\prime}$ & $5^{\prime}$ \\
\hline $\begin{array}{l}\text { S. faecium } \\
\text { Ha D2 } \\
\text { Ha D4 } \\
\text { Ha D6 } \\
\text { Ha D9 } \\
\text { Ha D10 }\end{array}$ & $\begin{array}{r}94 \\
114 \\
98 \\
50 \\
99\end{array}$ & $\begin{array}{r}93 \\
105 \\
111 \\
69 \\
91\end{array}$ & $\begin{array}{l}90 \\
77 \\
85 \\
93 \\
87\end{array}$ & $\begin{array}{l}96 \\
77 \\
93 \\
88 \\
78\end{array}$ & $\begin{array}{l}93 \\
57 \\
82 \\
90\end{array}$ & $\begin{array}{l}97 \\
64 \\
81 \\
93 \\
77\end{array}$ & $\begin{array}{r}103 \\
74 \\
89 \\
102\end{array}$ & $\begin{array}{r}101 \\
83 \\
87 \\
106 \\
98\end{array}$ \\
\hline $\begin{array}{l}\text { S. faecalis var } \\
\text { faecalis } \\
\text { Ha D7 } \\
\text { Ha D11 }\end{array}$ & $\begin{array}{r}91 \\
101\end{array}$ & $\begin{array}{r}119 \\
96\end{array}$ & $\begin{array}{r}122 \\
95\end{array}$ & $\begin{array}{r}104 \\
92\end{array}$ & $\begin{array}{l}121 \\
100\end{array}$ & $\begin{array}{r}107 \\
98\end{array}$ & $\begin{array}{r}99 \\
105\end{array}$ & $\begin{array}{l}102 \\
106\end{array}$ \\
\hline $\begin{array}{l}\text { S. faecalis var } \\
\text { liquefaciens } \\
\text { Ha D3 } \\
\text { Ha D5 } \\
\text { Ha D8 } \\
\text { Ha D13 }\end{array}$ & $\begin{array}{l}61 \\
49 \\
90 \\
84\end{array}$ & $\begin{array}{l}\frac{75}{98} \\
84\end{array}$ & $\begin{array}{l}86 \\
84 \\
58 \\
78\end{array}$ & $\begin{array}{r}\frac{98}{43} \\
103\end{array}$ & $\begin{array}{l}81 \\
87 \\
47 \\
73\end{array}$ & $\begin{array}{r}108 \\
66 \\
42 \\
78\end{array}$ & $\begin{array}{r}94 \\
104 \\
81 \\
93\end{array}$ & $\begin{array}{r}111 \\
119 \\
97 \\
75\end{array}$ \\
\hline $\begin{array}{l}\text { S. faecalis var } \\
\text { zymogenes } \\
\text { Ha } 12\end{array}$ & 82 & 96 & 106 & 101 & 97 & 98 & 91 & 98 \\
\hline
\end{tabular}

— Glc. : Glucose ; Lac. : Lactose.

- Le premier glucide correspond au milieu de culture et le second à l'essai de prise.

- The first sugar corresponds to the culture medium and the second one to the amino acid uptake. 
TABLEAU V

Pourcentage de prise de l'acide glutamique chez divers streptocoques en présence de lactose ou de glucose avec adaptation préalable au lactose ou au glucose

Percent of acid glutamic uptake by varied streptococci adapted on lactose or glucose using lactose or glucose as energy source

\begin{tabular}{|c|c|c|c|c|c|c|c|c|}
\hline \multirow{2}{*}{ Souche } & \multicolumn{2}{|c|}{ Glc.Lac./Glc.Glc. } & \multicolumn{2}{|c|}{ Lac.Glc./Glc.Glc. } & \multicolumn{2}{|c|}{ Lac.Lac./Glc.Glc. } & \multicolumn{2}{|c|}{ Lac.Lac./Lac.Glc } \\
\hline & $1^{\prime}$ & 5 & $1^{\prime}$ & $5^{\prime}$ & $1^{\prime}$ & $5^{\prime}$ & $1^{\prime}$ & $5^{\prime}$ \\
\hline $\begin{array}{l}\text { S. milleri } \\
\text { IP } 5625\end{array}$ & 34 & 53 & 77 & 82 & 44 & 53 & 57 & 65 \\
\hline $\begin{array}{l}\text { S. milleri } \\
\text { IP } 5426\end{array}$ & 52 & 61 & 97 & 94 & 81 & 67 & 83 & 69 \\
\hline $\begin{array}{l}\text { S. agalactiae } \\
\text { IP } 55118\end{array}$ & 58 & 75 & 84 & 87 & 66 & 71 & 78 & 81 \\
\hline $\begin{array}{l}\text { S. bovis } \\
\text { IP } 5623\end{array}$ & 52 & 61 & 124 & 120 & 105 & 86 & 85 & 72 \\
\hline $\begin{array}{l}\text { S. sanguis } \\
\text { IP } 55128\end{array}$ & 90 & 88 & 92 & 99 & 107 & 80 & 116 & 123 \\
\hline
\end{tabular}

- Glc. : Glucose ; Lac. : Lactose.

- Le premier glucide correspond au milieu de culture et le second à l'essai de prise.

- The first sugar corresponds to the culture medium and the second one to the amino acid uptake. 
pour la prise de l'acide glutamique, à savoir $S$. thermophilus et dans une moindre mesure $S$. salivarius.

Dans le second groupe sont classés les streptocoques fécaux et $S$. sanguis IP 55128 qui présentent peu de différences pour les deux substrats.

Le troisième groupe contient les streptocoques qui préfèrent le glucose. Pour certains l'adaptation préalable au lactose entraîne une bonne croissance et une prise améliorée de l'acide aminé en présence du diholoside, c'est le cas notamment des streptocoques du groupe N. Pour d'autres l'adaptation préalable n'a pas d'influence. C'est le cas de $S$. milleri, $S$. bovis, $S$. agalactiae et quelques streptocoques fécaux ( $S$. faecalis var. liquefaciens $\mathrm{HaD} 3$ et $\mathrm{HaD} 5$ et $S$. faecium HaD9).

La préférence du lactose par $S$. thermophilus et du glucose par les streptocoques du groupe $\mathrm{N}$ semble être étroitement liée au mode de transport des glucides.

Les quelques études réalisées sur l'utilisation du lactose par $S$. thermophilus ont montré que ce substrat est hydrolysé par une $\beta$-galactosidase (Tinson et al., 1982 ; Greenberg et Mahoney, 1982). De plus, la phosphorylation à travers la membrane n'existerait pas et la présence d'une phospho- $\beta$-galactosidase est douteuse. Ce glucide serait donc transporté par un système actif de type perméase dont l'énergie est fournie par l'ATP. Ceci explique sans doute la facilité de son utilisation. Au contraire, le glucose est transporté à l'intérieur des cellules de $S$. thermophilus par un système plus lent.

Il est certain que les streptocoques du groupe $\mathrm{N}$ possèdent une activité PEP-PTS spécifique du lactose (Mc KaY et al., 1969 ; THOMPson et Thomas, 1977 ; Thompson, 1978 ; Thompson et al., 1978 ; Thompson, 1979). En plus du PEP-PTS, ces micro-organismes peuvent transporter le glucose par le système perméase ATP-dépendant (Thompson, 1980). Par ailleurs, nous avons montré que ce groupe de bactéries utilise préférentiellement ce monosaccharide qui serait pris alors par un système perméase. Le retard d'utilisation du lactose est assimilé à l'induction d'enzymes nécessaires au mécanisme du PEP-PTS.

Le métabolisme des glucides chez les streptocoques fécaux a été très peu abordé. Heller et Röschenthaler (1978) ont décrit chez S. faecalis un système hydrolysant le lactose étroitement apparenté à celui du groupe $\mathrm{N}$ puisqu'il est constitué d'une phospho- $\beta$-galactosidase. Nous avons constaté qu'en général ces bactéries ne montrent pas de préférence vis-à-vis de l'un ou l'autre substrat, on peut formuler l'hypothèse que les glucides sont transportés par le même système.

D'autres espèces bactériennes, $S$. milleri, $S$. bovis et $S$. agalactiae, possèderaient des systèmes de transport similaires à ceux des streptocoques du groupe $N$.

$S$. salivarius se rapprocherait de $S$. thermophilus. Bien que ces deux espèces soient physiologiquement et biochimiquement distinctes, FARrow et CoLIINS (1984) proposent de classer $S$. thermophilus comme $S$. salivarius subp thermophilus; ces auteurs ont en effet montré qu'il existe une forte homologie dans la composition de l'ADN et des chaînes d'acides gras chez les deux bactéries. Dans notre étude, nous avons pu constater une préférence pour le lactose que nous retrouvons dans les courbes de croissance et la prise de l'acide glutamique chez les deux espèces. 


\section{Conclusion}

Du fait de l'importance de l'acide glutamique et des glucides dans le métabolisme des streptocoques lactiques et afin de caractériser les bactéries du genre Streptococcus, nous avons effectué cette étude qui nous a permis d'éclaircir certains points :

- 1e lactose est une source d'énergie efficace aussi bien pour la croissance que pour la perméation de l'acide glutamique chez l'espèce $S$. thermophilus ;

- au contraire, bien que les streptocoques du groupe $\mathrm{N}$ soient parfaitement adaptés au lait et donc au lactose, ils ne peuvent utiliser convenablement ce diholoside que s'ils y ont été adaptés au préalable;

- les streptocoques fécaux ne montrent pas de préférence pour le glucose ou le lactose, ni même une influence de l'adaptation à l'un ou l'autre substrat; - S. salivarius serait classé dans le même groupe que $S$. thermophilus.

Reçu le 18-7-1985.

Accepté pour publication le 4-11-1985

\section{Références bibliographiques}

AKPEMADO M., 1981. Etude du transport de la L-Valine, L-Leucine et L-Isoleucine chez Streptococcus thermophilus CNRZ 302. Thèse de troisième cycle, Nancy 1, $78 \mathrm{p}$.

AKPEMADO M., BRACoUART P., 1983. Uptake of branched-chain amino acids by Streptococcus thermophilus. Appl. Environ. Microbiol., 45, 136-140.

BissetT D.L., ANDERSON R.L., 1974. Genetic evidence for the physiological significance of the D-tagatose 6-phosphate pathway of lactose and D-galactose degradation in Staphylococcus aureus. J. Bacteriol., 119, 698-704.

Boos W., 1974. Bacterial transport Ann. Rev. Biochem., 43, 123-146.

BRACQUART P., 1981. An agar medium for the differential enumeration of Streptococcus thermophilus and Lactobacillus bulgaricus in yoghurt. J. Appl. Bacteriol., 51, 303-305.

BracoUart P., 1985. Etude des besoins en acides aminés et leur perméation chez Streptococcus thermophilus. Thèse de Docteur ès sciences, Nancy 1, $166 \mathrm{p}$.

DeIbel R.H., Seeley H.W., 1974. Streptococcaceae fam. nov. In : Bergey's manual of manual of determinative bacteriology, R.E. Gibbons, N.E. Baltimore, Williams and Wilkins.

FARRow J.A.E., Collins M.D., 1984. DNA base composition, DNA-DNA homology and long chain fatty acid on Streptococcus thermophilus and Streptococcus salivarius. J. Gen. Microbiol., 130, 137-362.

GREENBERG N.A., MAHONEY R.R., 1982. Production and characterization of $\beta$-galactosidase from Streptococcus thermophilus. J. Food Sci., 47, 1824-1828.

HelleR K., RöschenthaleR R., 1978, $\beta$-D-phosphogalactoside galactohydrolase of Streptococcus faecalis and the inhibition of its synthesis by glucose. Can. J. Microbiol., 24, 512-519.

Hemme D., WaHL D., NARdi M., 1980 a. Variations de l'équipement enzymatique de Streptococcus thermophilus. Lait, 60, (593-594), 111-129.

Hemme D., Nardi M., JeTte D., 1980 b. $\beta$-galactosidases et P- $\beta$-galactosidases de Streptococcus thermophilus. Lait, 60, (599-600), 595-618.

KASHKET E.R., WiLson T.H., 1972 a. Role of metabolic energy in the transport of $\beta$-galactosides by Streptococcus lactis. J. Bacteriol., 109, 784-789.

KASHKET E.R., WILSON T.H., 1972 b. Galactoside accumulation associated with ion movements in Streptococcus lactis. Biochem. Biophys. Res. Commun., 49, 615-620.

Kashket E.R., Blanchard A.G., Metzger W.C., 1980. Proton motrice force during growth of Streptococcus lactis cells. J. Bacteriol., 143, 128-134. 
KASHKet E.R., 1981. Proton motrice force in growing Streptococcus lactis and Staphylococcus aureus cells under aerobic and anaerobic conditions. J. Bacteriol., 146, 369-376.

Kundig W., Ghosh S., Roseman S., 1964. Phosphate bound to histidine in protein as an intermediate in a novel Phospho-Transferase system. Proc. Natl. Acad. Sci., 52, 1067-1074.

LaW B.A., SezGin E., Sharpe M.E., 1976. Amino acid nutrition of commercial cheese starters in relation to their growth in peptone supplemented whey media. J. Dairy Res., 43, 291-300.

Law B.A., Sharpe M.E., 1978. Streptococci in the Dairy Industry. In : "Steptococci", edited by Skinner F.A. and Quesnel L.B., 263-278.

Lawrence R.C., Thomas T.D., Terzaghi B.E., 1976. Reviews in the progress of dairy sciences : cheese starters. J. Dairy Res., 43, 141-193.

LeE R., Molskness T., SANDine W.E., Elliker P.R., 1973. Carbohydrate metabolism in lactic streptococci. Fate of galactose supplied in free or disaccharide form. Appl. Microbiol., 26, 951-958.

LEE D.R., 1974. Carbohydrate metabolism in lactic Streptococci with special reference to galactose. Diss. Abstr. Int, 34, 3615-3616.

Mc Kay L.L., WaLter L.A., SANDINE W.E., Elitiker P.R., 1969. Involvement of Phosphoenolpyruvate in Lactose Utilisation by group N Streptococci. I. Bacteriol., 99, 603-610.

Mc Kay L., Miller A., Sandine W.E., Elliker P.R., 1970. Mechanisms of Lactose Utilisation by lactic acid Streptococci : Enzymatic and Genetic Analyses. J. Bacteriol., 102, 804-809.

Mc KAY L.L., SANDine W.E., Elliker P.R., 1971. Lactose utilisation by lactic acid bacteria : a review. Dairy Sci. Abstr., 33, (7), 493-499.

Mitus O.E., Thomas T.D., 1981. Nitrogen sources for growth of lactic Streptococci in milk. N.Z.J. Dairy Sci. Technol., 16, 43-55.

Mitchell P., 1966. Chemiosmotic coupling in oxidative and photosynthetic phosphorylation. Biol. Rev., 41, 445-502.

Moran J.W., 1980. Branched-chain amino acid transport in Streptococcus agalactiae. Appl. Environ. Microbiol., 40, 25-31.

O'LeARY V.S., Woxchick J.H., 1976. Utilisation of lactose, glucose and galactose by a mixed culture of Streptococcus thermophilus and Lactobacillus bulgaricus in milk treated with lactase enzyme. Appl. Environ. Microbiol., 32, 89-94.

Rice G.H., Stewart F.H.C., Hiller A.J., Jago G.R., 1978. The uptake of amino acids and peptides by Streptococcus lactis. J. Dairy Res., 45, 93-107.

Somkuti G.A., STEInBerg D.H., 1979. Adaptation of Streptococcus thermophilus to lactose, glucose and galactose. J. Food Prot., 42, (11), 885-887.

Thomas T.D., TuRner K.W., CRow V.L., 1980. Galactose fermentation by Streptococcus lactis and Streptococcus cremoris : Pathways, Products and regulation. J. Bacteriol., 144, $672-682$.

Thomas T.D., CRow V.L., 1983. Lactose and sucrose utilisation by Streptococcus thermophilus. FEMS Microbiol. Lett., 17, 13-17.

Thompson J., Thomas T.D., 1977. Phosphoenolpyruvate and 2-phosphoglycerate : endogenous energy source for sugar accumulation by starved cells of Streptococcus lactis. J. Bacteriol., 130, 583-595.

THOMPson J., 1978, In vivo regulation of glycolysis and characterization of sugar : phosphotransferase systems in Streptococcus lactis, J. Bacteriol, 136, 465-476.

THOMPSON J., TURNER K.W., THOMAS T.D., 1978. Catabolite inhibition and sequential metabolism of sugars by Streptococcus lactis. J. Bacteriol., 133, 1163-1174.

THompson J., 1979. Lactose metabolism in Streptococcus lactis : Phosphorylation of galactose and glucose moieties in vivo. J. Bacteriol., 140, 774-785.

THOMPSON J., 1980. Galactose transport systems in Streptococcus lactis. J. Bacteriol., 144, 683-691.

THOMPSON J., Chassy M.B., 1981. Uptake and metabolism of sucrose by Streptococcus lactis. I. Bacteriol., 147, 543-551.

Tinson W., Hillier A.J., JAGo G.R., 1982. Metabolism of Streptococcus thermophilus. I, Utilization of lactose, glucose and galactose. Aust. I. Dairy Technot., 37, 8-13. 